\title{
Deposición Transversal de líquido de las Boquillas de Doble Abanico TTJ60-11004 y TTJ60-11002 EN Distintas Condiciones OPERACIONALES ${ }^{1}$
}

\author{
The Transversal Spray Deposition of Double Plain Spurt Nozzles TTJ60-11004 and TTJ60-11002 \\ in Different Operational Conditions
}
VIANA, R.G. ${ }^{2}$, FERREIRA, L.R. ${ }^{3}$, ROSELL, J.R. ${ }^{4}$, SOLANELLES, F. ${ }^{5}$, PLANAS, S. ${ }^{4}$, MACHADO, M.S. ${ }^{6}$ y MACHADO, A.F.L. ${ }^{7}$

\begin{abstract}
RESUMEN - El objetivo de este trabajo fue el de evaluar la deposición transversal de caldo de las boquillas pulverizadoras de doble abanico TTJ60-11004 y TTJ60-11002 en distintas condiciones operacionales. Se utilizaron 5 muestras de cada boquilla pulverizadora siendo considerada cada unidad, una repetición. La distribución de caldo fue evaluada por medio de una mesa de evaluación de distribución construida de acuerdo con la norma ISO 56821. Se evaluó el perfil de distribución individual, la distribución volumétrica simulada de la superposición de los chorros por medio del coeficiente de variación $(\mathrm{CV} \%)$ de los volúmenes colectados, el caudal y el ángulo de abertura de los chorros. Las condiciones operacionales fueron: presión de trabajo de 200, 300 y $400 \mathrm{Kpa}$, altura de 30, 40 y $50 \mathrm{~cm}$ en relación al blanco y espaciamiento entre boquillas simulados en Software (Microsoft Excel) entre 45 y $100 \mathrm{~cm}$. Las boquillas presentaron perfil individual descontinuo con la mayor deposición de líquido en la región central y reducción del volumen gradual en dirección a las extremidades. El aumento de la presión promovió alargamiento del perfil y de la franja de aplicación. Las boquillas proporcionaron perfil uniforme que dependió del espaciamiento entre las boquillas, con valores menores con reducción en el espaciamiento y en presiones mayores. El caudal y el ángulo del chorro aumentaron con el incremento en la presión.
\end{abstract}

Palabras-clave: boquilla pulverizadora, barra de pulverización, cobertura del blanco, herbicida de contacto.

\begin{abstract}
The objective of this work was evaluate the transversal spray deposition of double plain spurt nozzles TTJ60-11004 and TTJ60-11002 in different operational conditions. Was used five samples of each nozzle and considerate a unite one repetition. The spray deposition was evaluate on a table of evaluation distribution constructed in accordance with norm ISO 5682-1. Was evaluate a single spray distribution, volumetric distribution of the overlapping spurts by means of the coefficient of variation (CV\%), outflow and angle of opening of the spurts. The operational conditions had been pressure of work of 200, 300 and $400 \mathrm{kPa}$, height of 30,40 and $50 \mathrm{~cm}$ in relation to the target and spacing between nozzles in 45 and $100 \mathrm{~cm}$ simulated in software (Microsoft Excel). The nozzles presented discontinous individual profile with higher liquid deposition in the central region and gradual volume reduction in direction to extremities. The increase in the pressure promoted along of the profile and the band of application. The nozzles provided profile uniform to depend on the space between nozzles, with lesser values with reduction in the space and higher pressures. The outflow and the angle of the spurt had increased with the increment in the pressure
\end{abstract}

Keywords: spray nozzle, spray boom, target coverage, contact herbicide.

1 Recebido para publicação em 14.4.2009 e na forma revisada em 5.6.2009.

2 Eng ${ }^{\circ}$-Agr ${ }^{\circ}$, M.Sc., Doutorando em Fitotecnia, Departamento de Fitotecnia, Universidade Federal de Viçosa - DFT/UFV, $36570-000$ Viçosa-MG, Bolsista CNPq, <rafaelgomesviana@yahoo.com.br>; ${ }^{3}$ Prof. Associado - DFT/UFV; ${ }^{4}$ Prof. Doctor Departament d'Enginyeria Agroforestal. Universitat de Lleida-España; ${ }^{5}$ Investigador Centre de Mecanitzaciò Agrària, Departament d'Agricultura, Alimentació i Acció Rural, Generalitat de Catalunya; ${ }^{6}$ Graduando do curso de Agronomia - UFV; ${ }^{7}$ Doutor em Fitotecnia - DFT/UFV, Viçosa-MG.

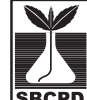

Planta Daninha, Viçosa-MG, v. 27, n. 2, p. 397-403, 2009 


\section{INTRODUCCIÓN}

Una de las maneras de maximizar la eficiencia de aplicaciones y reducir los riesgos de contaminación por fitosanitarios consiste en utilizar unas técnicas de aplicación adecuadas a cada situación, que tengan en cuenta las interacciones de factores climáticos, biológicos y económicos y que sean eficientes, con un mínimo impacto, tanto para el medio ambiente como para los cultivos sensibles (Matuo, 2001).

Gran parte de las aplicaciones de fitosanitarios son realizadas por medio hidráulico, o sea, utilizándose agua como diluente y vehículo del ingrediente activo hasta el blanco biológico. Su gran utilización se debe a la flexibilidad y facilidad de utilización en los medios productivos (Teixeira, 1997).

Las características de mayor interés en las aplicaciones hidráulicas son proporcionadas por las boquillas, siendo de gran importancia conocer las peculiaridades de cada una, a fin de seleccionar la mejor para cada ocasión (Miler \& Ellis, 2000; Cunha 2004). La deposición de caldo de manera eficiente, segura y económica en las aplicaciones hidráulicas sólo es posible cuando se dispone de boquillas que produzcan distribución de caldo y gotas de tamaño y número uniforme.

La distribución transversal de caldo es una evaluación que cuantifica si la distribución del producto fitosanitario sobre el blanco es uniforme y si se está depositando la cantidad realmente recomendada del producto. Su información es muy importante ya que permite determinar la distancia entre boquillas, así como la altura y la presión de trabajo ideal, para seleccionar de esta manera las condiciones que garanticen una deposición uniforme con las mejores condiciones de rendimiento operacional.

La evaluación es realizada por medio del coeficiente de variación del volumen acumulado en la superposición de los chorros de las boquillas en una barra (Bauer \& Raetano, 2004) y su valor, de acuerdo con la norma ISO 5682-2 (ISO, 1996), es del 7\% en presión y altura indicada por el fabricante ó $9 \%$ en condiciones distintas de éstas.

Valores superiores del recomendado pueden indicar falta o exceso de deposición de caldo entre las boquillas, proporcionando un control insuficiente del blanco biológico, toxicidad de los cultivos, pérdidas económicas y daño al medio ambiente (Cordeiro, 2001).

Para aplicaciones de herbicidas, la boquilla debe ser seleccionada, principalmente, en función del tipo de absorción y translocación del herbicida en la planta. Para aplicaciones de herbicidas de contacto y de translocación por xilema es necesaria una gran cobertura de las malas hierbas debido a su modo de acción. Productos de contacto como paraquat, diquat y lactofen son absorbidos por las hojas y rápidamente son metabolizados, provocando daños a la planta generalmente alrededor de la zona donde la gota se depositó. Productos de translocación por xilema, cuando son aplicados en post-emergencia, sólo tienen movimiento acrópeta, y, por lo tanto, también necesitan gran cobertura del blanco para un efectivo control (Silva et al., 2007).

Los ejemplos de aplicaciones de herbicidas que necesitan gran cobertura de malas hierbas, son aplicaciones de atrazine en post-emergencia en cultivos de maíz, paraquat y diquat en caña de azúcar, desecación antes de la sembradura directa y sobre los cultivos de granos para anticipar la cosecha. Otra aplicación que necesita gran cobertura es la transposición de herbicidas en cobertura muerta en los cultivos de siembra directa (Costa et al., 2004).

Por lo tanto, es necesario aumentar la cobertura de las malas hierbas y de cultivos blancos de manera que sea eficiente. Según, Courshee (1967), a medida que se aumenta el caudal y se reduce el tamaño de las gotas, se aumenta la cobertura del blanco, si bien estos factores pueden aumentar los riesgos de deriva de gotas y reducir la capacidad operacional del tratamiento.

El riesgo de deriva de herbicidas tiene como consecuencia una pérdida de control, impactos ambientales, daños para la salud del trabajador y a cultivos no blancos. Fitotoxidad en cultivos debido a deriva de herbicidas no selectivos fue observado por Rigoli et al. (2008) en plántulas de remolacha y zanahoria; Figueredo et al. (2007) en tomate y Tuffi Santos et al. (2007) han observado daños en la producción de madera en eucaliptos. 
Con el objetivo de incrementar la cobertura del blanco biológico, reducir la deriva de gotas y garantizar un mejor rendimiento operacional fueron desarrolladas las boquillas de doble abanico adjunto al sistema de impacto, el cual tiene un gran ángulo de abertura del chorro y gotas que abarcan desde diámetros medios hasta extremamente grandes (Spraying Systems, 2007). Viana et al. (2008) observaron porcentajes de cobertura mayor en las hojas inferiores de soja cuando utilizaron la boquilla de doble abanico TJ60-8002, en comparación con boquillas de chorro plano y cónico, toda vez que también proporcionaron una cobertura homogénea en toda la planta.

Las condiciones de trabajo de las boquillas de doble abanico TTJ60, que proporcionan una distribución de caldo uniforme y mejores rendimientos operacionales, no son conocidas, por lo tanto, es necesario su evaluación.

El objetivo de este trabajo fue evaluar la distribución de caldo de la boquilla TTJ6011004 Y TTJ60-11002 en distintas condiciones operacionales.

\section{MATERIAL Y MÉTODOS}

Los ensayos fueron realizados en los laboratorios del "Centre de Mecanitzaciò Agrària de la Genaralitat de Catalunya”. En los análisis se utilizaron cinco boquillas TTJ6011004 y TTJ60-11002 nuevas, siendo considerada para cada unidad una repetición.

El análisis de la distribución transversal de caldo fue realizado utilizándose una barra sobre un banco de ensayos para boquillas hidráulicas, compuesto por canales separados entre sí cinco centímetros y probetas con volumen de $400 \mathrm{ml}$ graduadas en $2 \mathrm{ml}$ para colectar el líquido de cada canal. La mesa fue diseñada siguiendo las recomendaciones de la norma ISO 5682-1 (ISO, 1996).

En el centro de la mesa se instaló una boquilla de manera que el chorro fuera pulverizado verticalmente. De cada boquilla se realizaron 3 medidas durante el tiempo suficiente para que, al menos, se completara el $90 \%$ del volumen de una probeta. El volumen

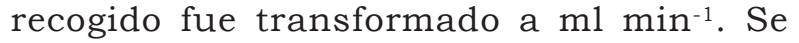
realizaron ensayos a presiones de 200, 300 y $400 \mathrm{kPa}$ y alturas de 30, 40 y $50 \mathrm{~cm}$ en relación a la superficie del banco.
A partir de los volúmenes medios colectados en las repeticiones, se determinaron los perfiles de distribución individuales mediante la posterior simulación en el ordenador (software: Microsoft Excel) del patrón medio de distribución volumétrica a lo largo de una barra de pulverización de $12 \mathrm{~m}$, así como la cuantificación del coeficiente de variación de los dos metros centrales. Fueron simulados espaciamientos entre boquillas de 45, 50, 60, $70,80,90$ y $100 \mathrm{~cm}$.

La medida del caudal fue realizada utilizándose un caudalímetro con análisis en tiempo real y con restricción de presión adjunta a un sistema hidráulico. Se analizaron las presiones de 200, 300 y $400 \mathrm{kPa}$.

El ángulo del chorro fue determinado por medio de imágenes frontales de las boquillas en funcionamiento, obtenidas mediante una cámara fotográfica digital con resolución de 6.1 megapixels, con la función flash activada. Las imágenes fueron analizadas en el ordenador con el software Image Tool 3.0. Los ángulos del chorro fueron medidos a partir de las proyecciones delimitadas tangencialmente a los bordes del chorro. Se cuantificó el ángulo izquierdo, derecho, total e índice de simetria, dado por la razón entre el ángulo derecho e izquierdo.

Los coeficientes de variación $(\mathrm{CV} \%)$ de la distribución de caldo fueron sometidos a estudios de análisis de regresión. Asimismo, el caudal y los ángulos de los chorros se sometieron a análisis de variancia y las medias fueron comparadas con el test de Tukey a $5 \%$ de probabilidad de error.

\section{RESULTADOS Y DISCUSIÓN}

El perfil proporcionado por las boquillas en todas las condiciones evaluadas fue estándar, con mayor acumulación de caldo en la región central y reducción de volumen en dirección a las extremidades (Figura 1). Estos perfiles de boquillas adyacentes se pueden fácilmente solapar proporcionando una distribución uniforme (FAO, 1996). Boquillas con este perfil son indicadas para aplicaciones en área total con superposición del chorro de otra boquilla y de esta manera, producir un perfil uniforme (Matuo, 2001).

Planta Daninha, Viçosa-MG, v. 27, n. 2, p. 397-403, 2009 

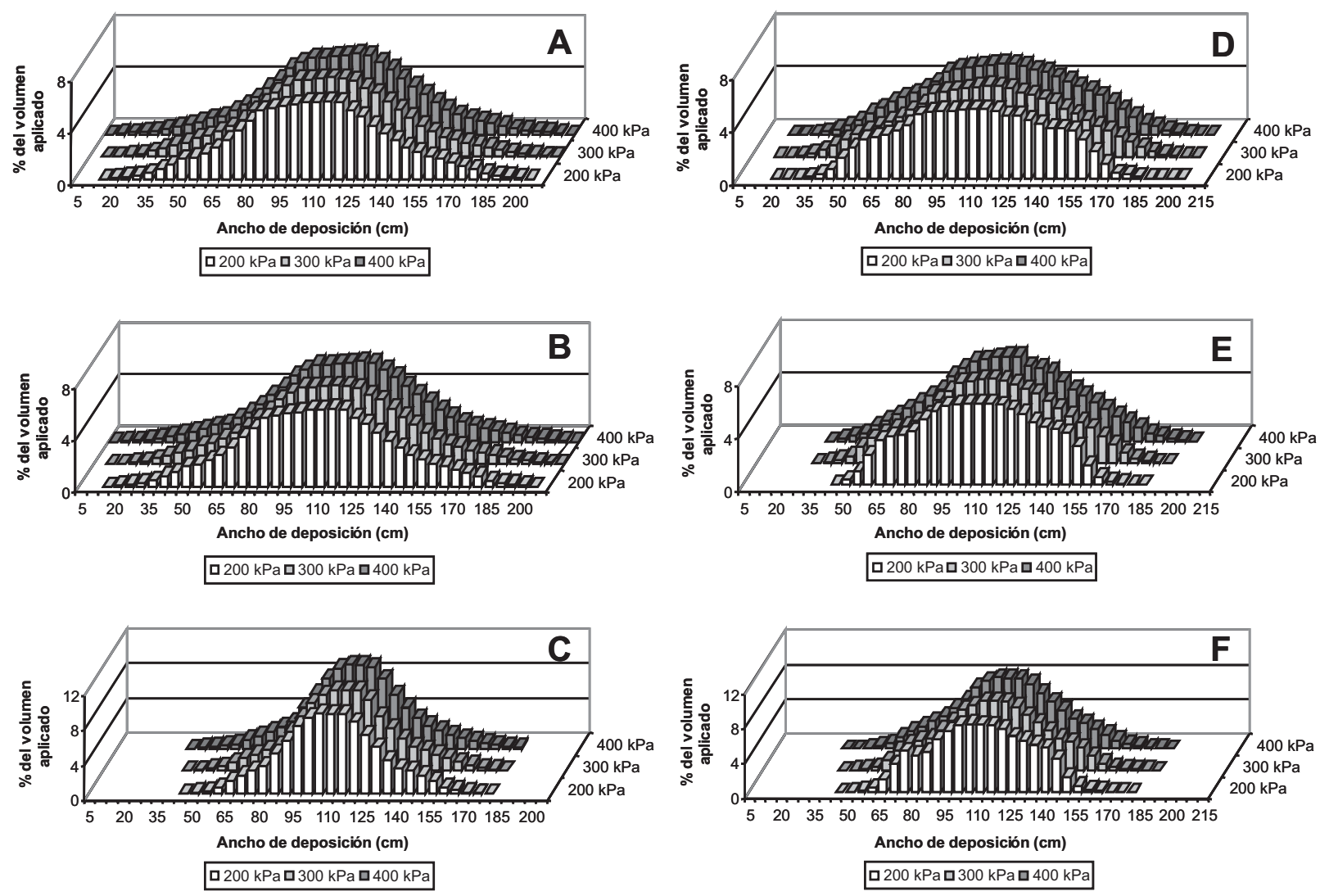

Figura 1 - Perfiles de distribución de líquido de las boquillas TTJ60-11004 a 50 (A), 40 (B) y $30 \mathrm{~cm}$ (C) de altura y TTJ60-11002 a 50 (D), 40 (E) y $30 \mathrm{~cm}$ de altura (F) en las presiones de 200, 300 y $400 \mathrm{kPa}$.

La boquilla TTJ60-11004, obtuvo mayor porcentaje de caldo en la región central, en comparación con la boquilla TTJ60-11002, siendo visible un pico de deposición en el centro (Figura 1A, B y C), lo que puede interferir en la distribución volumétrica en espaciamientos más anchos. Este factor puede ser explicado debido al tamaño de las gotas proporcionadas por la boquilla de mayor caudal. Según el fabricante, la boquilla TTJ60-11004 tiene mayor tamaño de gotas en comparación a la boquilla TTJ60-11002. Dado que las gotas mayores tienen menor energía cinética para ser transportadas a una mayor distancia, éstas quedan más próximas a la salida del chorro. Según Teixeira (1997), cuanto menor es el tamaño de las gotas mayor será el ancho de distribución transversal de caldo.

Alteraciones del perfil utilizando la misma boquilla pero con caudal distinto fueron observados por Cunha et al. (2004) utilizando las boquillas API110-02 y API110-04.
El ancho de aplicación fue aumentado con el incremento de la presión y de la altura de trabajo (Figura 1), debido al aumento en el ángulo total del chorro (Tabla 1). Comportamientos semejantes fueron observados por diversos autores evaluando la distribución de caldo en boquillas de chorro plano (Viana et al., 2007; Freitas et al., 2005; Cunha et al., 2006; Ferreira et al., 2007). Incrementos en el ancho de pulverización pueden ser beneficiosos en dos situaciones prácticas. En alturas mayores se puede alargar la distancia entre boquillas y así reducir el volumen de caldo y la distancia a ser pulverizada, aumentando el rendimiento operacional. La otra situación práctica consiste en disminuir la altura de la barra, reducir el espaciamiento entre boquillas y reducir los problemas de deriva de gotas.

Los valores del Coeficiente de variación (CV\%) en función del espaciamiento entre boquillas y altura de la barra en relación al blanco pueden ser visualizadas en la Figura 2. 
Tabla 1 - Medias de ángulo del chorro en el lado izquierdo, derecho, total e índice de simetría en las presiones de 200, 300 y $400 \mathrm{kPa}$

\begin{tabular}{|c|c|c|c|c|c|c|c|c|}
\hline \multirow{3}{*}{$\begin{array}{c}\text { Presión } \\
(\mathrm{kPa})\end{array}$} & \multicolumn{8}{|c|}{ Ángulo del chorro $\left({ }^{\circ}\right)^{*}$} \\
\hline & \multicolumn{2}{|c|}{ Izquierdo } & \multicolumn{2}{|c|}{ Derecho } & \multicolumn{2}{|c|}{ Total } & \multicolumn{2}{|c|}{ IS } \\
\hline & TTJ60 11004 & TTJ60 11002 & TTJ60 11004 & TTJ60 11002 & TTJ60 11004 & TTJ60 11002 & TTJ60 11004 & TTJ60 11002 \\
\hline 200 & $59,90 \mathrm{Ab}$ & $54,39 \mathrm{Bc}$ & $60,18 \mathrm{Ab}$ & $55,00 \mathrm{Bc}$ & $120,08 \mathrm{Ac}$ & $109,39 \mathrm{Bc}$ & 1,00 & 1,01 \\
\hline 300 & $62,59 \mathrm{Aa}$ & $59,61 \mathrm{Bc}$ & $63,69 \mathrm{Ab}$ & $60,10 \mathrm{Bb}$ & $126,28 \mathrm{Ab}$ & $119,71 \mathrm{Bb}$ & 1,01 & 1,00 \\
\hline 400 & $64,00 \mathrm{Aa}$ & $62,98 \mathrm{Ba}$ & $64,86 \mathrm{Aa}$ & $63,03 \mathrm{Ba}$ & $128,86 \mathrm{Aa}$ & $126,01 \mathrm{Ba}$ & 1,01 & 1,00 \\
\hline
\end{tabular}

* Medias seguidas de las mismas letras mayúsculas, en la línea, y minúsculas, en la columna, no son distintas a 5\% de probabilidad por el test de Tukey. Medias sin letras no son significativas entre sí por el test $\mathrm{F}$ a $5 \%$ de probabilidad.

Las boquillas experimentaron un aumento del $\mathrm{CV} \%$ con el incremento del espaciamiento entre éstas, pero en algunas situaciones este crecimiento fue lineal o cuadrático, dependiendo de la presión de trabajo y de la altura de la pulverización.

La boquilla TTJ60-11004 obtuvo un aumento cuadrático del CV\% en la altura de $50 \mathrm{~cm}$ (Figura 2A) y lineal en las alturas de 40 y $30 \mathrm{~cm}$ (Figura 2B y C). En la altura de $50 \mathrm{~cm}$ se obtuvieron espaciamientos con deposición uniforme entre $45 \mathrm{~cm}$ y 78,$93 ; 76,02$ y 74 , $67 \mathrm{~cm}$ en las presiones de 200, 300 y $400 \mathrm{kPa}$, respectivamente (Figura 2A). A $40 \mathrm{~cm}$ de altura se obtuvieron espaciamientos con deposición uniforme entre $45 \mathrm{~cm}$ y 56,$28 ; 54,16$ y $53 \mathrm{~cm}$, en las presiones de 200, 300 y $400 \mathrm{kPa}$ respectivamente (Figura 2B). Finalmente, a $30 \mathrm{~cm}$ de altura se obtuvieron espaciamientos adecuados sólo en las presiones de $200 \mathrm{kPa}$, entre 45 y $46 \mathrm{~cm}$ entre boquillas y a $400 \mathrm{kPa}$, entre 45 y $48 \mathrm{~cm}$ (Figura 2C).

La boquilla TTJ60-11002 obtuvo un aumento cuadrático del CV\% en la altura de $50 \mathrm{~cm}$, en la presión de $200 \mathrm{kPa}$ lineal para las presiones de 300 y $400 \mathrm{kPa}$ (Figura 2D) en $40 \mathrm{~cm}$ de altura, aumento lineal en la presión de $200 \mathrm{kPa}$ y cuadrático para 300 y $400 \mathrm{kPa}$ (Figura 2E). Para $30 \mathrm{~cm}$, en todas las presiones, el aumento fue lineal (Figura 2F).

En la altura de $50 \mathrm{~cm}$, para la boquilla TTJ60-11002 se obtuvieron espaciamientos con deposición uniforme entre 45 y $52 \mathrm{~cm} ; 100$ y $100 \mathrm{~cm}$ en las presiones de 200, 300 y $400 \mathrm{kPa}$, respectivamente (Figura 2D), siendo los espaciamientos de $100 \mathrm{~cm}$ de gran interés práctico debido al mayor rendimiento operacional y a que las barras de aplicación tienen, tradicionalmente, un espaciamiento de $50 \mathrm{~cm}$ entre boquillas, de modo que fácilmente, se puede utilizar esta configuración simplemente cerrando una boquilla a cada $100 \mathrm{~cm}$. A $40 \mathrm{~cm}$ de altura se obtuvieron espaciamientos con deposición uniforme entre $45 \mathrm{~cm}$ y 53,24 y $82,76 \mathrm{~cm}$ en las presiones de 200, $300 \mathrm{y}$ $400 \mathrm{kPa}$ respectivamente (Figura 2F). Ya en $30 \mathrm{~cm}$ de altura, se obtuvieron espaciamientos adecuados sólo en las presiones de 300 y $400 \mathrm{kPa}$ con espaciamiento entre 45 y $57,88 \mathrm{~cm}$ para presión de $300 \mathrm{kPa}$ y 45 y 63,41 $\mathrm{cm}$ para la presión de $400 \mathrm{kPa}$ (Figura 2F). Las alturas de 30 y $40 \mathrm{~cm}$ son ideales para reducir la deriva de gotas y pueden ser utilizadas para este fin, según se ha analizado.

Freitas et al. (2005) han observado un comportamiento análogo utilizando la boquilla de impacto TT11002 y han indicado su uso para reducir la deriva de gotas en la altura de $30 \mathrm{~cm}$ con espaciamientos entre boquillas de $50 \mathrm{~cm}$ o también para aumentar el rendimiento operacional con espaciamiento de hasta $120 \mathrm{~cm}$ y altura de $50 \mathrm{~cm}$.

El ángulo del chorro (Tabla 1) y el caudal (Tabla 2) aumentaron al incrementarse la presión. La boquilla TTJ60-11004 obtuvo mayor ángulo izquierdo, derecho y total que la boquilla TTJ60-11002. Este hecho presentó, como consecuencia, un ancho de pulverización más largo (Figura 1) pero con distribución en el blanco de manera distinta que la boquilla TTJ60-11002.

Los ángulos son simétricos, como se puede visualizar en la Tabla 1, siendo éste un factor de extrema importancia para una distribución uniforme. Valores equivalentes fueron observados por Viana et al. (2007) y Cunha et al. (2004) con boquillas de chorro plano. 

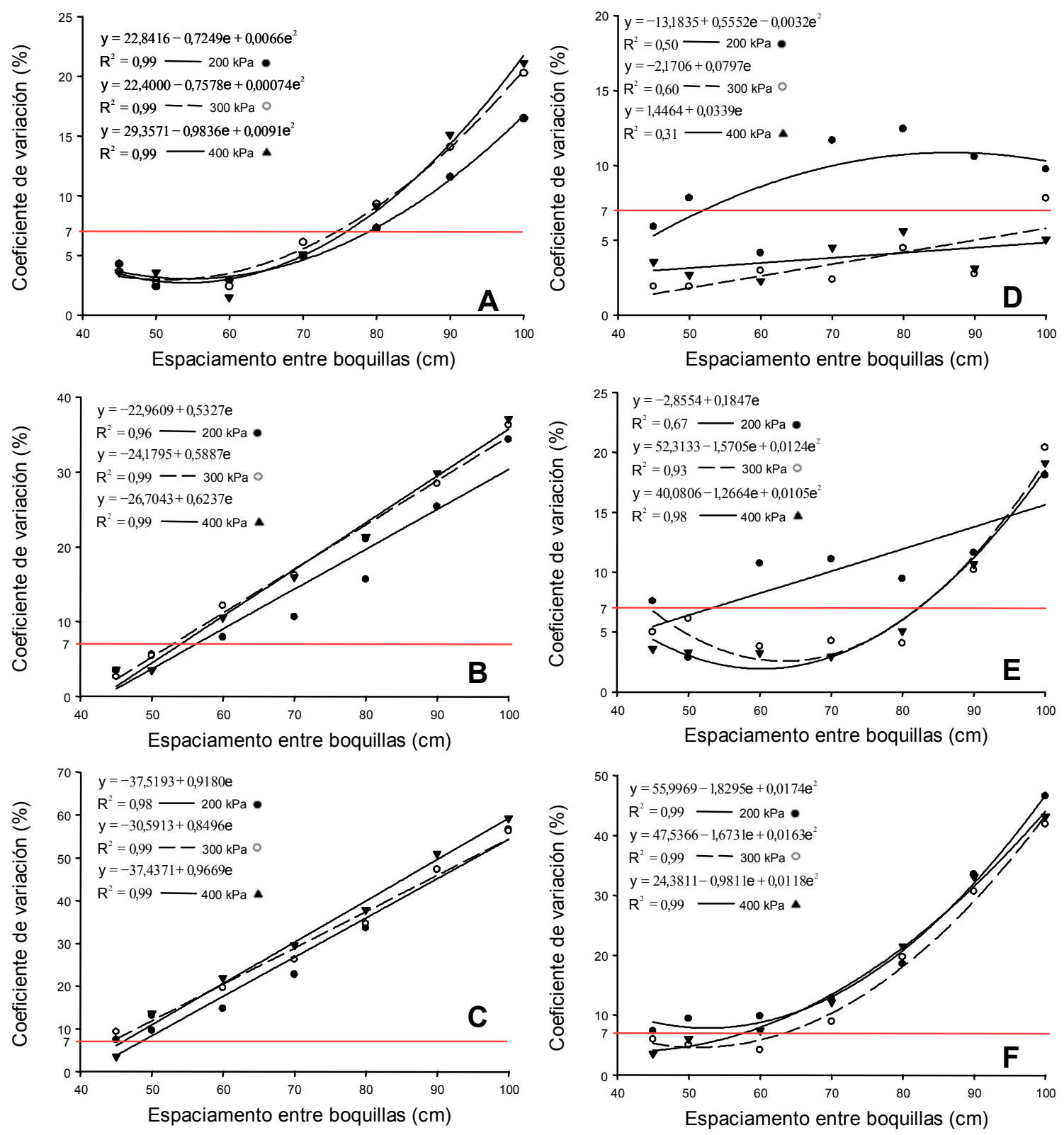

Figura 2 - Uniformidad de distribución volumétrica en función de los espaciamientos entre boquillas de las puntas TTJ6011004 en las alturas de 50 (A), 40 (B) y $30 \mathrm{~cm}$ (C) y TTJ60-11002 en las alturas de 50 (D), 40 (E) y $30 \mathrm{~cm}$ (F) en las presiones de 200, 300 y $400 \mathrm{kPa}$.

Tabla 2 - Medias de caudal de las boquillas TTJ60-11004 y TTJ60-11002 en las presiones de 200, 300 y $400 \mathrm{kPa}$

\begin{tabular}{|c|c|c|}
\hline \multirow{2}{*}{ Presión $(\mathrm{kPa})$} & \multicolumn{2}{|c|}{ Caudal $\left(\mathrm{L} \mathrm{min}^{-1}\right)^{*}$} \\
\cline { 2 - 3 } & TTJ60 11004 & TTJ60 11002 \\
\hline 200 & $1,29 \mathrm{~A} \mathrm{c}$ & $0,65 \mathrm{~B} \mathrm{c}$ \\
\hline 300 & $1,60 \mathrm{~A} \mathrm{~b}$ & $0,80 \mathrm{~B} \mathrm{~b}$ \\
\hline 400 & $1,80 \mathrm{~A} \mathrm{a}$ & $0,92 \mathrm{~B} \mathrm{a}$ \\
\hline
\end{tabular}

* Medias seguidas de las mismas letras mayúsculas, en la línea, y minúsculas, en la columna, no son distintas a $5 \%$ de probabilidad por el test de Tukey.
El caudal de las boquillas a $300 \mathrm{kPa}$ está de acuerdo con las normas de clasificación Visiflo (ECS, 1997) en color rojo para la boquilla TTJ60-11004 y amarillo para la boquilla TTJ6011002 (Tabla 2). El caudal de la boquilla TTJ6011004 es más grande que el de la boquilla TTJ60-11002 y por eso deben ser utilizadas de acuerdo con las características de la aplicación, como velocidad de aplicación y cobertura necesaria del blanco biológico. 
Las boquillas ensayadas presentan posibilidades de uso para aumentar el rendimiento operacional, con espaciamientos mayores, o para reducir problemas de deriva de gotas con reducción de la altura de pulverización y espaciamiento entre boquillas. Tanto el ángulo de pulverización como el caudal aumentaron con el aumento de la presión.

\section{AGRADECIMIENTOS}

Al "Conselho Nacional de Desenvolvimento Científico e Tecnológico (CNPq)", por otorgarle una beca de estudio doctoral al primer autor. A la "Coordenação de Aperfeiçoamento de Pessoal de Nivel Superior (CAPES)", por otorgar una beca de estudios en el exterior. A "Spray Systems CO.”, por donar las boquillas para los ensayos.

\section{LITERATURA CITADA}

BAUER, F. C.; RAETANO, C. G. Distribuição volumétrica de calda produzida pelas pontas de pulverização XR, TP e TJ sob diferentes condições operacionais. Planta Daninha, v. 22, n. 2, p. 275-284, 2004.

CORDEIRO, A. M. C. Como a tecnologia de aplicação de produtos fitossanitários pode contribuir para o controle de pragas, doenças e plantas daninhas. In: ZAMBOLIM, L. Manejo integrado: fitossanidade, cultivo protegido, pivô central e plantio direto. Viçosa, MG: Universidade Federal de Viçosa, 2001. p. 683-721.

COSTA, A. G. F. et al. Dinâmica da transposição de herbicida através de palha de aveia-preta utilizando diferentes pontas de pulverização. Planta Daninha, v. 22, n. 4, p. 561-566, 2004.

COURSHEE, R. J. Application and use of foliar fungicides. TORGESON, D. C. (Ed.). Fungicide- An advance treatise. New York: Academic Press, 1967. p. 239-286.

CUNHA, J. P. A. R. et al. Uniformidade de liquido por bicos de pulverização hidráulicos de jato plano e de jato cônico vazio. Eng. Agric., v. 12, n. 3, p. 192-202, 2004.

CUNHA, J. P. A. R.; RUAS, R. A. A. Uniformidade de distribuição de pontas de pulverização de jato plano duplo com indução de ar. Pesq. Agropec. Trop., v. 36, n. 1, p. 61-66, 2006.

EUROPEAN COMMITTEE FOR STANDARDIZATION ECS. Agricultural and forestry machinery - Sprayers and liquid fertilizer distributors - Environmental protection Part 2: Low crop sprayers - prEN 12761-2: 1997. Brussels: 1997. $17 \mathrm{p}$.
ORGANIZACIÓN DE LAS NACIONES UNIDAS PARA LA AGRICULTURA Y LA ALIMENTACIÓN - FAO. Equipo portátil de aplicación de pesticidas para uso en agricultura de servicios. Rome: 1996. v. 1.151 p.

FERREIRA, M. C. et al. Fatores qualitativos da ponta de energia hidráulica ADGA 110015 para pulverização agrícola. Eng. Agríc., v. 27, n. 2, p. 471-478, 2007.

FIGUEREDO, S. S. et al. Influência de doses reduzidas do Glyphosate no tomateiro (Lycopersicon esculentum Mill.). Planta Daninha, v. 25, n. 3, p. 849-857, 2007.

FREITAS, F. C. L. et al. Distribuição volumétrica de pontas de pulverização turbo teejet 11002 em diferentes condições operacionais. Planta Daninha, v. 23, n. 1, p. 161-167, 2005.

\section{INTERNATIONAL ORGANIZATION FOR}

STANDARDIZATION - ISO. Equipment for crop protection - Spraying equipment - Part 2: test methods for agricultural sprayers. Geneva: 1996. 13 p. (ISO 5682/1).

MATUO, T. et al. Tecnologia de aplicação e equipamentos. In: Curso de proteção de plantas. Módulo 2. Brasília, DF: ABEAS; Viçosa, MG: UFV, 2001. 85 p.

MILLER, P. C. H.; ELLIS M. C. B. Efects of formulation on spray nozzle performance for applications from ground-based boom sprayers. Crop Protec., v. 19, p. 609-615, 2000.

RIGOLI, R. P. et al. Resposta de plantas de beterraba (Beta vulgaris) e de cenoura (Daucus carota) à deriva simulada de glyphosate. Planta Daninha, v. 26, n. 2, p. 451-456, 2008.

SILVA, A. A. et al. Métodos de controle de plantas daninhas. In: SILVA, A. A.; SILVA, J. F. (Eds). Tópicos em manejo de plantas daninhas. Viçosa, MG: Universidade Federal de Viçosa, 2007. p. 17-55.

SPRAYING SYSTEMS CO. Productos de pulverización para la agricultura - Catálogo 50A-E-SP/P. 2007.

TEIXEIRA, M. M. Influencia del volumen de caldo y de la uniformidad de distribución transversal sobre la eficacia de la pulverización hidráulica. 1997. 310 f. Tesis (Doctorado en Agronomía) - Escuela Técnica Superior de Ingenieros Agrónomos, Universidad Politécnica de Madrid, Madrid, 1997.

TUFFI SANTOS, L. D. et al. Crescimento do Eucalipto sob efeito da deriva de glyphosate. Planta Daninha, v. 25, n. 1, p. 133-137, 2007.

VIANA, R. G. et al. Deposição de gotas no dossel da soja por diferentes pontas de pulverização hidráulica e pressões de trabalho. Eng. Agric., v. 16, n. 4, p. 428-435, 2008.

VIANA, R. G. et al. Características técnicas de pontas de pulverização LA-1JC e SR-1. Planta Daninha, v. 25, n. 1, p. 211-218, 2007.

Planta Daninha, Viçosa-MG, v. 27, n. 2, p. 397-403, 2009 